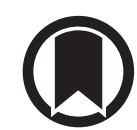

CrossMark

\section{Ventilation-perfusion SPECT versus CTPA in young adult females with suspected pulmonary embolism}

\author{
To the Editor:
}

We read with interest the "2019 ESC Guidelines for the diagnosis and management of acute pulmonary embolism" developed by the European Society for Cardiology (ESC) in collaboration with the European Respiratory Society [1]. An important question addressed in the 2019 ESC Guidelines, which is also discussed in the recently updated guideline on ventilation-perfusion (VQ) scintigraphy from the European Association of Nuclear Medicine (EANM) [2], is whether computed tomography pulmonary angiography (CTPA) or VQ scintigraphy should be the diagnostic method of choice in suspected pulmonary embolism. This quandary is notably pertinent in young female adults who are at an increased risk of pulmonary embolism due to oral contraceptive use or pregnancy, and we would like to add to the discussion in relation to this patient group by raising specific points relating to the diagnostic performance and radiation exposure of VQ scintigraphy versus CTPA.

The 2019 ESC Guidelines provide specific recommendations regarding planar VQ scintigraphy, which is typically based on six consecutive static projections, and interpreted according to a widely criticised probabilistic diagnostic classification. The lower diagnostic accuracy compared to CTPA, as well as an unacceptably high rate of non-diagnostic scans, has undoubtedly contributed to the tarnished reputation of VQ scintigraphy. However, planar VQ scintigraphy has largely been abandoned throughout Europe and is no longer recommended by EANM [2]. It has been replaced by the single photon emission computed tomography (SPECT)-based technique mentioned in the 2019 ESC Guidelines, which provides three-dimensional images of the pulmonary VQ distribution. VQ SPECT is interpreted according to a trinary classification as 1) showing evidence of pulmonary embolism, 2) showing no evidence of pulmonary embolism, or 3) as non-diagnostic. We acknowledge several of the methodological limitations of the studies on the accuracy of VQ SPECT that are addressed in the 2019 ESC Guidelines, notably their retrospective design and the inclusion of VQ SPECT itself in the reference standard in some studies, as well as the paucity of outcome studies. Furthermore, as also mentioned, the optimal scanning technique, particularly the added clinical value of supplementing VQ SPECT with computed tomography (CT) remains to be defined in various categories of patients, but low-dose CT (without i.v. contrast), which increases the specificity of VQ SPECT while also providing information regarding the same range of differential diagnoses as CTPA, is currently the most widely used modality $[2,3]$. We do, however, respectfully disagree with the notion in the 2019 ESC Guidelines that the criteria for diagnosing acute pulmonary embolism on VQ SPECT and their use in clinical practice are inconsistent. Since the previous EANM guideline on VQ SPECT from 2009, the consensus diagnostic criteria for acute pulmonary embolism has been the presence of at least one segmental or two subsegmental mismatched perfusion defects that conform with pulmonary vascular anatomy [4], and we are not aware of any European nuclear medicine facilities that currently use alternative definitions. With this approach, and while keeping the methodological limitations mentioned above in mind, the diagnostic performance of VQ SPECT is comparable to and may even exceed that of CTPA, while the non-diagnostic rates are similar $(<5 \%)[2,3,5]$.

In terms of radiation exposure, the high rate of cell proliferation in the breast tissue of young females renders this tissue notably radiation sensitive. The risk of radiation-induced cancer is proportional to radiation dose [6], and according to a previous simulation-based study performed a decade ago, one case of radiation-induced breast cancer would develop for every 330 CTPA scans performed in 20-year old

@ERSpublications

Ventilation-perfusion SPECT should be preferred over CTPA in young adult females with suspected pulmonary embolism https://bit.ly/2XYWdJ7

Cite this article as: Hansen SL, de Nijs R, Mortensen J, et al. Ventilation-perfusion SPECT versus CTPA in young adult females with suspected pulmonary embolism. Eur Respir J 2020; 55: 2000448 [https://doi. org/10.1183/13993003.00448-2020]. 
TABLE 1 Effective and absorbed doses in different imaging modalities for diagnosing pulmonary embolism

\begin{tabular}{|c|c|c|}
\hline Modality & Effective dose mSv & $\begin{array}{l}\text { Absorbed dose in } \\
\text { breast tissue mGy }\end{array}$ \\
\hline CTPA (standard pulmonary embolism protocol) & 1.9 & 5.4 \\
\hline CTPA (dual energy pulmonary embolism protocol) & 4.3 & 11.9 \\
\hline Perfusion scintigraphy $\left({ }^{99 \mathrm{~m}} \mathrm{Tc}-\mathrm{MAA}\right)$ & $0.44-1.32$ & $0.20-0.60$ \\
\hline Ventilation scintigraphy $\left({ }^{99 m}\right.$ Tc-DTPA) & $0.14-0.21$ & $0.038-0.057$ \\
\hline Ventilation scintigraphy ( $\left.{ }^{99 m} \mathrm{Tc}-T e c h n e g a s\right)$ & $0.30-0.45$ & $0.13-0.20$ \\
\hline Ventilation scintigraphy $\left({ }^{81 \mathrm{~m}} \mathrm{Kr}\right)$ & $0.028-0.28$ & $0.0037-0.037$ \\
\hline Low-dose CT of chest (without i.v. contrast) & 1.5 & 3.3 \\
\hline
\end{tabular}

Ventilation-perfusion single photon emission computed tomography (SPECT) involves ventilation scintigraphy with either ${ }^{99 \mathrm{~m}} \mathrm{Tc}$-labelled DTPA (aerosol), ${ }^{99 \mathrm{~m}} \mathrm{Tc}$-Technegas or ${ }^{81 \mathrm{~m}} \mathrm{Kr}$, and a perfusion scintigraphy using ${ }^{99 \mathrm{~m} T c-l a b e l l e d ~ m a c r o a g g r e g a t e d ~ a l b u m i n ~(M A A), ~ a s ~ w e l l ~ a s ~ o p t i o n a l ~ l o w-d o s e ~ c o m p u t e d ~}$ tomography (CT). The scintigraphy-based doses are calculated from established dose coefficients and the standard administered activities in our institution, which are in accordance with current European Association of Nuclear Medicine guidelines [2, 8, 9], where breast dose calculations for ${ }^{81 \mathrm{~m}} \mathrm{Kr}$ were calculated by scaling lung doses with their conversion factors (S values) [9]. CT doses are weighted values based on measurements on a standard CT dose index (CTDI) phantom, using an ion chamber placed consecutively in the centre, as well as in four locations in the periphery of the phantom, and were found to agree with the measured CTDI values reported by the scanner in all instances. The effective dose and absorbed dose in breast tissue were calculated from CTDI based on Monte Carlo simulations using CTexpo software (SASCRAD, Buchholz, Germany). \#: in the case of X-rays and gamma radiation, the absorbed dose in breast tissue has the same numerical value as the equivalent dose with the unit mSv. CTPA: computed tomography pulmonary angiography.

females [7]. Considerable progress in CT-technology has been made over the past years, aiming at both improving image quality and reducing radiation dose, but the difference in radiation exposure associated with VQ SPECT and CTPA nonetheless remains substantial. However, the difference in radiation dose to the breast tissue is largely underestimated when considering the effective (whole-body) doses alone. According to current data obtained from two state of the art CTPA protocols available at our institution, the effective doses of the CTPA protocols are only a few times higher than that of our VQ SPECT protocol, but the absorbed dose in breast tissue is nonetheless 18-40 times higher (table 1). Even if the VQ SPECT is supplemented with a low-dose CT, both the effective and absorbed dose remains less than that of CTPA (table 1).

On basis of the above, we posit that VQ SPECT should be considered the first-choice imaging modality in young adult females with suspected acute pulmonary embolism when a nuclear medicine facility is available. We furthermore suggest that specific recommendations regarding planar VQ scintigraphy are omitted from future clinical guidelines on the diagnosis and management of acute pulmonary embolism.

Sofie Lindskov Hansen ${ }^{1}$, Robin de Nijs ${ }^{1}$, Jann Mortensen ${ }^{1,2,3}$ and Ronan M.G. Berg $\circledast^{1,4,5}$

${ }^{1}$ Dept of Clinical Physiology, Nuclear Medicine and PET, Rigshospitalet, University Hospital of Copenhagen, Copenhagen, Denmark. ${ }^{2}$ Dept of Clinical Medicine, University of Copenhagen, Copenhagen, Denmark. ${ }^{3}$ Dept of Medicine, The National Hospital, Torshavn, Faroe Islands. ${ }^{4}$ Dept of Biomedical Sciences, Faculty of Health and Medical Sciences, University of Copenhagen, Copenhagen, Denmark. ${ }^{5}$ Centre for Physical Activity Research, Rigshospitalet, Copenhagen, Denmark.

Correspondence: Ronan M.G. Berg, Dept of Biomedical Sciences, Faculty of Health and Medical Sciences, University of Copenhagen, Blegdamsvej 3, DK-2200 Copenhagen, Denmark. E-mail: ronan@sund.ku.dk

Received: 26 Feb 2020 | Accepted after revision: 15 April 2020

Conflict of interest: None declared.

\section{References}

1 Konstantinides SV, Meyer G, Becattini C, et al. 2019 ESC Guidelines for the diagnosis and management of acute pulmonary embolism. Eur Respir J 2019; 54: 1901647.

2 Bajc M, Schümichen C, Grüning T, et al. EANM guideline for ventilation/perfusion single-photon emission computed tomography (SPECT) for diagnosis of pulmonary embolism and beyond. Eur J Nucl Med Mol Imaging 2019; 46: 2429-2451.

3 Mortensen J, Gutte H. SPECT/CT and pulmonary embolism. Eur J Nucl Med Mol Imaging 2014; 41: S81-S90. 

clinical considerations for diagnosis of pulmonary emboli with V/P(SPECT) and MDCT. Eur J Nucl Med Mol Imaging 2009; 36: 1528-1538.

5 Hess S, Frary EC, Gerke O, et al. State-of-the-art imaging in pulmonary embolism: ventilation/perfusion single-photon emission computed tomography versus computed tomography angiography - controversies, results, and recommendations from a systematic review. Semin Thromb Hemost 2016; 42: 833-845.

6 Valentin J. Low-dose extrapolation of radiation-related cancer risk. Ann ICRP 2005; 35: 1-142.

7 Smith-Bindman R, Lipson J, Marcus R, et al. Radiation dose associated with common computed tomography examinations and the associated lifetime attributable risk of cancer. Arch Intern Med 2009; 169: 2078-2086.

8 Mattsson S, Johansson L, Leide Svegborn S, et al. Radiation dose to patients from radiopharmaceuticals: a compendium of current information related to frequently used substances. Ann ICRP 2015; 44: 7-321.

9 Task Group of Committee 2. Radiation dose to patients from radiopharmaceuticals. Ann ICRP 1987; 18: 1-29.

\title{
The optimal imaging test for diagnosis of acute pulmonary embolism: a second chance for lung scintigraphy?
}

\author{
From the author:
}

S.L. Hansen and colleagues present a number of arguments aiming to improve the "tarnished" (as the authors call it) reputation of ventilation-perfusion (VQ) scintigraphy, and to promote single photon emission computed tomography (VQ SPECT) in the diagnostic work-up of suspected pulmonary embolism. Among others, the authors provide a table showing the effective and absorbed doses in different imaging modalities used for the diagnosis or exclusion of pulmonary embolism. This information complements and extends the data provided in the 2019 European Society of Cardiology (ESC) guidelines for the diagnosis and management of acute pulmonary embolism, developed in collaboration with the European Respiratory Society (ERS) [1]. In agreement with these guidelines, S.L. Hansen and colleagues point to the significantly lower radiation dose absorbed by the breast tissue during VQ SPECT as opposed to computed tomography pulmonary angiography (CTPA). In view of these differences, the authors advocate the use of VQ SPECT as first-line diagnostic modality in young adult females with suspected pulmonary embolism.

The first update of the ESC guidelines for pulmonary embolism, including formal recommendations, was published in 2008 [2], replacing a more narrative earlier version [3]. The most important novelty of the update was the revised diagnostic algorithm for patients with suspected pulmonary embolism, in which CTPA replaced VQ scintigraphy (and invasive pulmonary angiography) as the new central imaging test and diagnostic "gold standard" [2]. The evidence for the shift to CTPA had been delivered a few years before by the results of major accuracy and management trials [4, 5]. The 2008 guidelines did acknowledge that the validity of the (planar) lung scan had been evaluated in several prospective clinical outcome studies, showing low event rates and suggesting that it is safe to withhold anticoagulant therapy in patients with a normal perfusion scan [2]. This fact had also been confirmed in a randomised trial comparing VQ scan with CTPA [6]. In parallel, it was mentioned [2] that the evolving SPECT technique promised to overcome the problem of frequent non-diagnostic intermediate probability planar scans $[7,8]$. However, in the second update of the guidelines 6 years later [9], and although VQ scan continued to be explicitly mentioned as a valid diagnostic test for pulmonary embolism, no compelling new evidence was cited to question the central position of CTPA, which had meanwhile conquered the vast majority of hospitals in

@ERSpublications

Ventilation-perfusion scintigraphy may continue to be the first-line diagnostic method for pulmonary embolism in institutions that can ensure its uninterrupted availability and the expertise in its interpretation https://bit.ly/2y3Dlhx

Cite this article as: Konstantinides SV. The optimal imaging test for diagnosis of acute pulmonary embolism: a second chance for lung scintigraphy?. Eur Respir J 2020; 55: 2001426 [https://doi.org/10.1183/ 13993003.01426-2020]. 
Europe and beyond. Specifically for VQ SPECT, with or without low-dose computed tomography, the conclusion of the guidelines was that large-scale prospective studies were needed to validate these new approaches [9]. Now, in the most recent 2019 update to which Hansen and co-workers refer, the wording and overall evaluation of VQ scintigraphy, including SPECT, remained largely unchanged [1].

Does this "stagnation" mean that the chapter of pulmonary embolism diagnosis should finally be considered closed? I agree with the authors that the answer is by no means so simple. Being experts in nuclear medicine, S.L. Hansen and colleagues eloquently defend the consistency of the diagnostic criteria for pulmonary embolism in VQ SPECT. One should also fairly admit that "large-scale" prospective trials required to provide high-level evidence in favour of VQ SPECT are hardly realistic in the era of CTPA dominance. In addition, if VQ SPECT is considered to be the technical evolution of planar VQ scan, its clinical evaluation may not need to be as extensive as would be required for a diagnostic method based on a totally new principle. The authors further emphasise the advantages of the technique, the strongest of which is the low dose of absorbed radiation compared to CTPA. In fact, their suggestion to consider VQ SPECT in the diagnostic work-up of young women is principally in line with the algorithm proposed by the 2019 ESC/ERS guidelines for suspected pulmonary embolism in pregnancy, in which (perfusion) scintigraphy assumes a clearly visible position as an alternative to CTPA [1]. S.L. Hansen and colleagues go further by proposing to extend these recommendations to VQ SPECT and broaden the target population to non-pregnant young women.

Overall, there is no fundamental disagreement between the arguments of Hansen and co-workers and the recommendations of the 2019 ESC/ERS guidelines. Except, perhaps for these few words in the concluding paragraph: "first-choice imaging modality". To issue a broad recommendation in favour of VQ SPECT and against the current gold standard CTPA, the guidelines must, even if this is only for a specific clinical setting, ensure that two conditions are met, assuming that the two methods are otherwise equivalent: 1) the alternative is broadly available, as is the standard; and 2) the alternative is always and rapidly available, as is (in most cases) the standard, so that diagnosis or exclusion of pulmonary embolism will be equally fast. Unfortunately, however, we have to admit that, in the majority of European hospitals, these conditions are not met for VQ SPECT. Pulmonary embolism is an acute, potentially life-threatening situation, and nothing can be more dangerous for patients, including young women, than delaying its confirmation or exclusion by several hours or even days until the test can be performed. Transferring the patient to another facility that can perform VQ SPECT is also not an acceptable option. Consequently, it is unlikely that the CTPA-based "main" algorithm will change in the near future. On the other hand, and as the authors point out, VQ scintigraphy may certainly become (or continue to be) the first-line diagnostic method in institutions that can ensure its uninterrupted availability and the expertise in its interpretation.

Stavros V. Konstantinides $\oplus^{1,2}$

${ }^{1}$ Center for Thrombosis and Hemostasis, University Medical Center of the Johannes Gutenberg University, Mainz, Germany. ${ }^{2}$ Dept of Cardiology, Democritus University of Thrace, Alexandroupolis, Greece.

Correspondence: Stavros V. Konstantinides, Centre for Thrombosis and Haemostasis, University Medical Centre Mainz, Langenbeckstrasse 1, Bldg. 403, 55131 Mainz, Germany. E-mail: stavros.konstantinides@unimedizin-mainz.de

Received: 27 April 2020 | Accepted after revision: 27 April 2020

Conflict of interest: S.V. Konstantinides reports grants and personal fees from Bayer AG, Daiichi Sankyo, BTG - Boston Scientific Group and Actelion - Janssen, grants from Boehringer Ingelheim and Servier, personal fees from Pfizer Bristol Myers Squibb and MSD, outside the submitted work.

Support statement: The work of Stavros Konstantinides was supported by the German Federal Ministry of Education and Research (BMBF 01EO1003 \& 01EO1503). The author is responsible for the contents of this publication. Funding information for this article has been deposited with the Crossref Funder Registry.

\section{References}

1 Konstantinides SV, Meyer G, Becattini C, et al. ESC Guidelines for the diagnosis and management of acute pulmonary embolism. Eur Respir J 2019; 54: 1901647.

2 Torbicki A, Perrier A, Konstantinides S, et al. Guidelines on the diagnosis and management of acute pulmonary embolism: the Task Force for the Diagnosis and Management of Acute Pulmonary Embolism of the European Society of Cardiology (ESC). Eur Heart J 2008; 29: 2276-2315.

3 Guidelines on diagnosis and management of acute Pulmonary Embolism. Task Force on Pulmonary Embolism, European Society of Cardiology. Eur Heart J 2000; 21: 1301-1336.

4 van Belle A, Buller HR, Huisman MV, et al. Effectiveness of managing suspected pulmonary embolism using an algorithm combining clinical probability, D-dimer testing, and computed tomography. JAMA 2006; 295: 172-179.

5 Perrier A, Roy PM, Sanchez O, et al. Multidetector-row computed tomography in suspected pulmonary embolism. N Engl J Med 2005; 352: 1760-1768. 
6 Anderson DR, Kahn SR, Rodger MA, et al. Computed tomographic pulmonary angiography vs ventilation-perfusion lung scanning in patients with suspected pulmonary embolism: a randomized controlled trial. JAMA 2007; 298: 2743-2753.

7 Reinartz P, Kaiser HJ, Wildberger JE, et al. SPECT imaging in the diagnosis of pulmonary embolism: automated detection of match and mismatch defects by means of image-processing techniques. J Nucl Med 2006; 47: 968-973.

8 Reinartz P, Wildberger JE, Schaefer W, et al. Tomographic imaging in the diagnosis of pulmonary embolism: a comparison between V/Q lung scintigraphy in SPECT technique and multislice spiral CT. J Nucl Med 2004; 45: 1501-1508.

9 Konstantinides SV, Torbicki A, Agnelli G, et al. ESC Guidelines on the diagnosis and management of acute pulmonary embolism: The Task Force for the Diagnosis and Management of Acute Pulmonary Embolism of the European Society of Cardiology (ESC). Eur Heart J 2014; 35: 3033-3073. 